examines the paradox, known as demographic transition.

One model proposed to explain the phenomenon holds that fewer offspring receive more resources, making them more likely to have offspring themselves. The model posits that richer people might have fewer children, but would ultimately have more descendents over subsequent generations.

Not so, say Anna Goodman of the London School of Hygiene and Tropical Medicine and her team. In their analysis of 14,000 Swedish people born between 1915 and 1929 and their descendents, small family size predicted greater socioeconomic success in children, grandchildren and great-grandchildren, particularly among families that already had high socioeconomic status. But small family size did not translate into greater reproductive success among the descendants.

Proc. R. Soc. B http://dx.doi. org/10.1098/rspb.2012.1415 (2012)

\section{BOTANY}

\section{Plants split cells to put down roots}

Plants cells cannot migrate, so plants control the development of multilayered tissues such as roots through asymmetric cell divisions that create layers with different identities and functions.

A team headed by Athanasius Marée of the John Innes Centre in Norwich, UK, and Ben Scheres at the University of Utrecht in the Netherlands unravelled the molecular pathway that regulates these cell divisions in the root tip. Stem cells in the model plant Arabidopsis are triggered to divide unevenly by a positive feedback loop that takes effect when a protein called RETINOBLASTOMA ceases to inhibit another, called SCARECROW. Gradients of a growth hormone and a protein called SHORT ROOT ensure that this loop is triggered in the correct place. Protein degradation during the division prevents the process from continuing indefinitely. Cell http://dx.doi.org/10.1016/ j.cell.2012.07.017 (2012)

\section{ASTROPHYSICS}

\section{Disintegrating planet spotted}

NASA's Kepler spacecraft seems to have spotted a distant, rocky planet that is falling apart.

Kepler hunts for planets beyond the Solar System by searching for steady, periodic dimming in the light of parent stars, which indicates the passage of an orbiting body. In the case of the star KIC 12557548 , however, the drop in starlight varies in strength with each passage. Scientists have suggested that this variability is a sign of an orbiting planet that is trailed by a large dust cloud.

Matteo Brogi of Leiden University in the Netherlands and his team modelled the dust cloud and found that its presence could indeed explain the Kepler data. The cloud is probably the result of the planet being bombarded by so much stellar radiation that it has begun breaking up into dust. Astron. Astrophys. http:// dx.doi.org/10.1051/00046361/201219762 (2012)

\section{BIOGEOSCIENCES \\ Pruning back carbon estimates}

Incorporating tree-height data into calculations of the amount of carbon stored in tropical forests reduces the estimates by roughly $13 \%$.

Ted Feldpausch of the University of Leeds, UK, and his team analysed data from 327 tropics-wide plots, as well as 20 sites where tropical trees have been cut down, collecting data on factors such as the weight and height of the trees, and their carbon density. The team found that information on tree height was crucial for making accurate biomass estimates, and that the relationship between height

COMMUNITY CHOICE

The most viewed papers in science

\title{
Hunter-gatherer workout disproved
}

\section{HIGHLY READ \\ on www.plosone.org in August}

For 11 days, Herman Pontzer of Hunter College in New York and his colleagues measured daily energy expenditure and physical activity levels in 30 adults from a Hadza huntergatherer group in Tanzania. Controlling for factors such as age, sex, body fat and body mass, the researchers compared their results to individual and population data from a spectrum of societies, including Western countries. Hadza individuals walk longer distances and forage for resources. So, unsurprisingly, they had higher physical-activity levels than Westerners. However, on average, both groups used the same amount of energy on a daily basis, as well as when walking or resting, suggesting that the rate of energy expenditure is an evolved trait that is independent of culture.

Obesity trends in Western populations could be unrelated to a sedentary lifestyle, the researchers suggest.

PLoS ONE 7, e40503 (2012) and carbon storage varied by region.

The authors underscore the importance of including better data in biomass maps, in which field measurements are increasingly being integrated with remote-sensing data to improve accuracy.

Biogeosciences 9, 3381-3403 (2012)

MATERIALS
Sticking the
unstickable

Researchers have succeeded in sticking together two supremely unsticky polymers - Teflon and cross-linked poly(dimethylsiloxane), the slippery coating used as backing paper for stickers.

The secret to their success lies in tetrapodal zinc oxide crystals: micrometre-scale structures (pictured) shaped rather like children's jacks. Strewing these between the polymers and heating the resulting sandwich to $100^{\circ} \mathrm{C}$ for 40 minutes creates a kind of 'micro/nano Velcro'. The polymers can be peeled apart

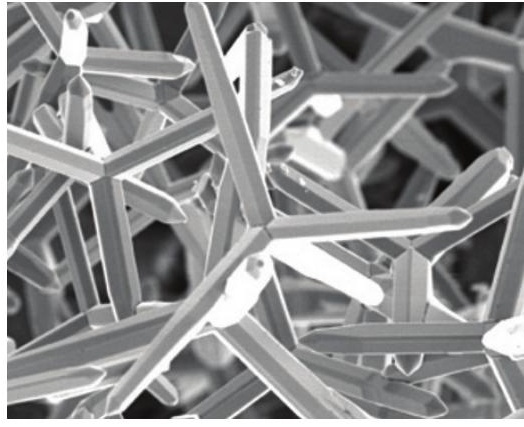

only by applying a force of about 200 Newtons per metre - more than that required to unstick Scotch tape.

Rainer Adelung and his team at the University of Kiel, Germany, did not stick the unstickable for glory alone. Stuck together, these surfaces will have applications in technologies such as membranes for separating liquids, and biomedical implants.

Adv. Mater. http://dx.doi. org/10.1002/adma.201201780 (2012)

\section{$\rightarrow$ NATURE.COM}

For the latest research published by Naturevisit:

www.nature.com/latestresearch 